\title{
On the Realization of Back-Propagation on a Transputer Based System
}

\author{
Petridis V. ${ }^{1}$, Adamidis P. ${ }^{1}$, Margaritis K.G. ${ }^{2}$ \\ ${ }^{1}$ Dept of Electrical Eng, Aristotle University of Thessaloniki, 54006 Greece. \\ Email: petridis@vergina.eng.auth.gr \\ 2 Informatics Centre, University of Macedonia, Greece
}

This paper presents some experimental results on the realization of a parallel simulation of an Artificial Neural Network (ANN) on a Transputer based system. The Back-Propagation algorithm has been used, and the parallelization technique utilized is the Processor Farm based on the partition of the training patterns between the available transputers so that each transputer (trainee) is trained on a different part of the training set, while another transputer (the controller) keeps track of the global network state.

Initially the controller sends to each trainee information on: network architecture, initial weights and a part of the training pattern set. Each trainee processes the patterns presented to it (forward flow, back propagation and weight update), and then it returns to the controller, the updated weights and the output error that it has computed. The controller computes the average error and the average of each weight received from each trainee. If the average error has not reached an acceptable level, then the controller sends the new weights and another part of the training pattern file to each trainee. This procedure continues, until the error reaches a specified level. Trainees update their connection weights each time they finish processing a training pattern, since it was found that this accelerates the convergence of the ANN.

The number of patterns sent to each trainee is kept constant and it is termed Update Rate, since the weight average is found after each trainee has finished processing its patterns.

The Telmat system used, is consisted of $2420 \mathrm{Mhz}$ T800 transputers with 2MB RAM. The software was developed in $\mathrm{C}$ under Helios, and Component Distribution Language (CDL).

The main issues addressed are: (a) the speed-up of the parallel simulation against the simulation on one processor and (b) the existence of an optimal value of the Update Rate

A number of simulations has been performed. Fig.1 shows the results for cartesian to polar transformations. The simulation on one Transputer requires 324 iterations and 14194 seconds.

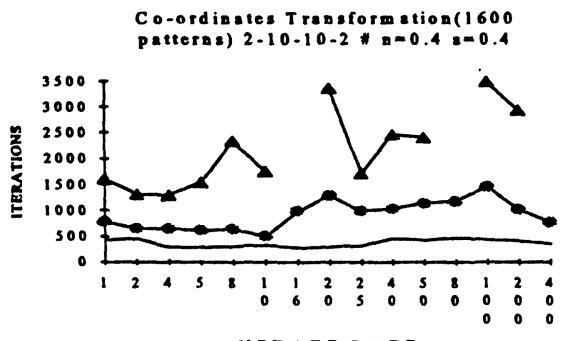

U PDATE RATE

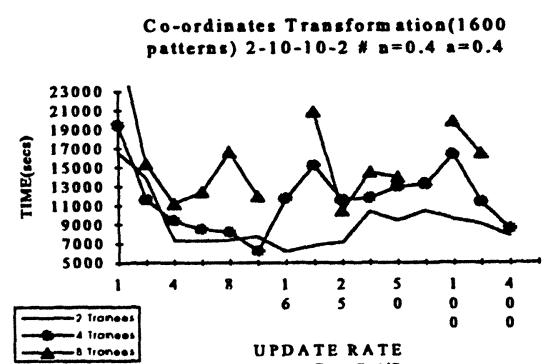

Co-ordinates Transformation(1600

Fig. 1 Performance of the Parallel ANN simulation trained on the functions $z=\left(x^{2}+y^{2}\right)^{1 / 2}$ and $\theta=\arctan (y / x)$.

\section{REFERENCES}

1. Smith L.S., "Neural net simulation using Transputer nets", SERC/DTI Transputer Loan Report, 1988.

2. Tollenaere $T$, Orban $G A$, " Simulating modular neural networks on message-passing multiprocessors", Parallel Computing 17, 1991.

3. Wang C, Wu C, "Parallel simulation of Neural Networks", Simulation, 56, 1991. 